\title{
Role of Entamoeba gingivalis in periodontitis: Myth or reality!
}

Ravikiran Reddy Vundela1, Vidya Sagar Sisnity², Raja Babu Palaparthi ${ }^{3}$, Vikram Reddy Guntakanla ${ }^{4}$

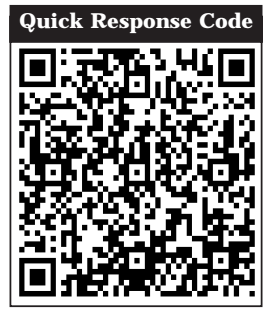

doi: $10.5866 / 2016.8 .10100$

${ }^{1}$ Post Graduate Student

2Professor

${ }^{3} \mathrm{HOD} \&$ Professor

${ }^{4}$ Reader

Department of Periodontics,

Kamineni Institute of Dental Sciences.

Narketpally, Nalgonda, Telangana.

\section{Article Info:}

Received: April 9, 2016

Review Completed: May 9, 2016

Accepted: J une 10, 2016

Available Online: September, 2016 (www.nacd.in)

(C) NAD, 2016 - All rights reserved

\section{Email for correspondence:}

ravikiranreddy.vundela@gmail.com

\begin{abstract}
:
Periodontitis is considered as a chronic inflammatory disease of tooth supporting structures. It is a multifactorial disease where interplay between putative periodontal pathogens, host factors, and environmental factors result in disease. Scope of oral microbiology is often reduced to oral bacteriology. Recent advances in microbiological diagnostics resulted in identification of nonbacterial microbial inhabitants of the oral cavity. Some of these microorganisms like viruses, archae and protozoa are considered to have periodontopathic roles. Though Entamoeba gingivalis was discovered in 1849 its role as periodontal pathogen is still controversial due to less number of studies and diagnostic limitations. This review aims to study the available literature on the role of Entamoeba gingivalis in periodontal disease.
\end{abstract}

Key words: Periodontitis, Microbiota, Oral hygiene, Entamoeba gingivalis, Virulence factors, Diagnosis.

\section{INTRODUCTION:}

Periodontitis is one of the most prevalent diseases worldwide. Unlike many other diseases, the etiology of periodontitis is not clearly determined. Literature on periodontitis focuses mainly on bacterial etiology and host response. However, this hypothesis fails to explain the difference between gingivitis and periodontitis in terms of etiology.

It has been estimated that approximately 700 distinct microbial phylotypes can be present as natural inhabitants of dental plaque. ${ }^{1}$ Any individual may harbor 150 or more different species. Scope of oral microbiology is often reduced to oral bacteriology. ${ }^{2}$ However; the oral cavity comprises a much more diverse microbiota than merely a bacterial one. Archaea, yeasts, protozoa, and viruses are the nonbacterial microorganisms that are found in plaque. Many of these species can reside in the oral cavity as commensals but they can also cause several oral diseases.

Viruses are important ulcerogenic and tumorigenic agents of the human mouth. Herpesviruses are present abundantly in inflamed gingival tissue, crevicular fluid, and subgingival plaque in periodontally diseased sites. Their role in the etiology of periodontitis has been suggested 
based on these findings and their ability to induce proinflammatory cytokines. ${ }^{3}$ However, there is no doubt that herpesviruses are present in the periodontal pocket but the hypothesis that they are involved in the etiology of periodontitis is still controversial. ${ }^{4}$

Many yeast species have been isolated from the oral cavity. The majority of isolates are Candida, and the most prevalent species is Candida albicans (C. albicans). C. albicans is a normal commensal of the oral cavity but also an opportunistic pathogen. In otherwise healthy individuals, oral candidosis rarely manifests in the gingiva. However, C. al bicans is frequently isolated from the subgingival flora of patients with severe periodontitis. ${ }^{5}$

Archae are single celled eukaryotes. Their role in oral diseases is only beginning to be explored. Methanogenic archae have been isolated from patients with periodontal disease. They mainly occur in the anerobic part of the subgingival biofilm and in infected root canals. ${ }^{6}$ Archaea were identified in 37 out of 48 samples from periodontal disease sites with the use of molecular detection methods. Also, they were not identified in periodontally healthy subjects. ${ }^{7}$

Protozoa are the other group of microorganisms that inhabit the oral cavity as commensals but have the potential to turn into opportunistic pathogens. Protozoa tend to beseen in cases of poor oral hygiene and poor periodontal health. Protozoa that induce local infections include Entamoeba gingivalis ( $E$. gingivalis) and Trichomonas tenax (T. tenax). The flagellate, $T$. tenax, is considered nonpathogenic and is common in $10 \%$ of the population, mainly related to poor oral hygiene. It is associated with periodontal disease and is abundant in cases of gum and tonsil diseases, but there is no evidence indicating their involvement in the etiology of these conditions. ${ }^{8} \mathrm{Up}$ to $95 \%$ of the population with poor oral hygiene may be infected with Entamoeba gingivalis.

\section{DISCUSSION:}

\section{E. gingivalis:}

It belongs to Eukaryota domain, Protozoa kingdom, Amoebozoa phylum, Archamoebae class, and Entamoeba genus. It is transmitted between Humans through saliva during oral contact or common usage of dishes and utensils. Gross (1849) identified it for the first time in human mouth. Though it was observed by Gross in patients with periodontitis its putative role as etiologic agent was not taken into consideration. Therefore its role was neglected until 1983 when Lyons detected E. gingivalis in periodontal pockets. He also reported that they were absent in healthy sites. Lyons called the infection of oral cavity caused by E. gingivalis as oral amoebiasis. Based on the assumption that E. gingivalis was responsible for periodontitis he recommended a therapeutic protocol adding oxygen peroxideand metronidazol e to periodontal therapy. ${ }^{9}$ This therapeutic protocol was proved to be effective, recently by Bonner et al. in 2013. ${ }^{10}$

\section{Incidence of E. gingivalis:}

Wantland \& Wantland studied the incidence of E. gingivalis and T. tenax in 700 subjects and reported that $39 \%$ harbor E. gingivalis; $23 \%$ harbored $\mathrm{T}$. tenax; and $17.7 \%$ harbored both. They also investigated the presence of organisms in relation to oral conditions. In relatively clean and healthy mouths (514 patients): E. gingivalis, 26.4\%; T. tenax, $11.2 \%$; both, $6.4 \%$; early progressive stages of periodontosis (141 patients): E. gingivalis, $65.2 \%$; T. tenax, 48.2\%; both, 39\%; advanced periodontosis (45 patients): E. gingivalis, 100\%; T. tenax, 80\%; both, $80 \%{ }^{11}$

Ozcelik et al. studied oral scraping samples from 220 patients. Oral protozoa were detected in 58 (26.4\%) of 220 samples. In 48 (21.8\%) of the positive 58 specimens, E. gingivalis was detected alone whereas T. tenax was present in only $2(1 \%)$ specimens. In 8(3.6\%) specimens E. gingivalis and T. tenax were identified together. Authors also reported that gum problems, presence of tartar, smoking habits, and brushing habits were found to be statistically significantly related with the presence of oral protozoa. ${ }^{12}$

Keyes \& Rams (1983) identified E. gingivalis in all 65 patients with destructive periodontal disease. They also reported that E. gingivalis was absent in subjects with marginal gingivitis and in 
excellent Periodontal health. ${ }^{13}$ In contrast, Dao et al. (1983) reported presence of E. gingivalis in disease free individuals. ${ }^{14}$

A study conducted on the incidence of $E$. gingivalis among 100 students of the Karol Marcinkowski University of Medical Sciences in Poznan showed that $72 \%$ of the students were infected with E. gingivalis and the incidence of the parasite, as well as the intensity of the infection, is correlated with oral hygiene status and the use of various preparations intended for maintaining oral cavity hygiene. ${ }^{15}$

Microbiologic analysis of 51 biofilm samples from patients diagnosed with gingivitis, periodontitis and healthy individuals revealed that 16 biofilm samples ( $50 \%$ were from patients with gingivitis and $50 \%$ were from patients with periodontitis) were positive for $\mathrm{E}$. gingivalis. It was not found in the biofilm samples of control group. Microbiologic analysis of salivary samples revealed that 18 samples were positive for $E$. gingival is $(61 \%$ were from patients with periodontitis and $39 \%$ were from patients with gingivitis) and it was not found in control group. The salivary $\mathrm{pH}$ of the participants ranged from 6.0 and 8.0 , and the likelihood of the microorganisms being present in the saliva decreased as salivary alkalinity increased. No statistically significant correlation was found between age and presence of microorganism. ${ }^{16}$

Despite the high incidence of $\mathrm{E}$. gingivalis and T. tenax in the dental biofilm there are only few reports on their role in the pathogenesis of periodontitis. Infection rate of E ntamoeba gingival is in patients with periodontitis is much higher than that of healthy patients. ${ }^{17}$ Chen et al inferred that E. gingivalis is related to periodontitis. ${ }^{18}$

An unsual case of $E$. gingivalis in acute osteomyelitis of mandible was recently reported. ${ }^{19}$

\section{Association with periodontitis:}

Entamoeba gingivalis, found only in the dental biofilm and saliva of patients with periodontal disease, corroborates the hypothesis that this protozoan might be involved in the development of periodontal disease, as has been suggested by other studies. ${ }^{20-22}$
It has been suggested that Entamoeba gingivalis could affect the formation of the dental biofilm and contribute to the development and progression of periodontal disease. ${ }^{23}$ Other researchers state that this microorganism may be opportunistic, since it is capable of proliferating in the microenvironment of the mucobuccal fold affected by periodontal disease. ${ }^{24}$ Therefore, if $\mathrm{E}$. gingivalis helps the development and progression of periodontal disease and periodontal disease increasingly facilitates the proliferation of these protozoa, this vicious circle could explain the increased incidence of these microorganisms in the dental biofilm of patients with gingivitis and periodontitis. A suspension of Entamoeba gingival is was spread on the gingival margins of rats immunosuppressed with prednisol one, leading to the development of the clinical signs of periodontal disease much faster than that observed in immunocompetent rats. ${ }^{25}$

Some authors injected E ntamoeba gingivalis in the base of the gingival pocket of rats, which induced periodontal abscess in 78.9\% (30/38) of sample. Live Entamoeba gingivalis were found in purulent secretions and cultured from the periodontal abscess. These results strongly suggest that infection by Entamoeba gingivalis may destroy the gingival tissues.

These data indicate that immunosuppression may play a role in the pathogenesis of periodontal disease induced by this commensal.

\section{Detection of E. gingivalis:}

Light microscopy, Polymerized chain reaction (PCR) and real-time PCR were used to detect E. gingivalis from saliva, gingival scrapings, and subgingival plaque samples.

\section{Light microscopy:}

For nearly 100 years, light microscopic studies have also demonstrated a high incidence of the protozoan parasite Entamoeba gingivalis in individuals suffering from oral disease including periodontitis. ${ }^{26}$ This has led to a speculation that it might also be a contributing factor to periodontal disease. However, the average prevalence of $\mathrm{E}$. 
gingivalis based on microscopic observations was $62 \%$.

\section{Polymerized chain reaction (PCR) analysis and Real-time PCR analysis:}

Trim et al (2011) compared the presence of E. gingival is in diseased periodontal sites, healthy sites in periodontitis patients and periodontally healthy individuals using conventional PCR and real-time PCR. For conventional PCR a previously described (Kikuta et al., 1996) primer set, small subunit ribosomal DNA (SSU rDNA), small subunit ribosomal RNA gene of $\mathrm{E}$. gingivalis was used. For real-time PCR assay a primer set was designed to amplify 135-bp fragment inside the SSU rDNA of E. gingivalis. Conventional PCR assay detected E. gingivalis in $27 \%$ of periodontal pockets while realtime PCR assay detected E. gingivalis in $69 \%$ of the periodontal pockets. NoE. gingivalis were detected in any of the healthy gingival pocket sites using either type of PCR assay. Their study results supported the concept that the presence of $E$. gingivalis is associated only with periodontal pockets. $^{27}$

\section{Virulence factors and interactions with putative periodontopathic bacteria:}

Studies addressing the virulence factors of $\mathrm{E}$. gingivalis are lacking. However, previous studies on other cl osely related species, Entamoeba hystolytica, have clearly identified virulence factors that contribute to and cause tissue destruction. Studies addressing potential interactions between periodontopathic bacteria and E. gingivalis are lacking. However, since both periodontopathic bacteria and $\mathrm{E}$. gingivalis occur in periodontal pockets some interactions would be expected to occur. Previous studies on other species of Entamoeba genus identified that they can serve as host cells for bacteria thus protecting the bacteria from detrimental environmental factors.

Results from an in vitro study showed that growth of the periodontopathic bacterium Aggregatibacter actinomycetemcomitans (previously called Actinobacillus actinomycetemcomitans) was affected by the presence of unidentified oral amoebae. The amoebae enhanced the growth of $A$. actinomycetemcomitans in media which otherwise failed to meet nutritional requirement. These bactereia were found within the vacuoles and around the amoebae. This interaction was considered important because bacteria harbored within the vacuoles of amoebae could gain protection from immune system and antibiotics given as part of periodontal therapy. Therefore the author suggested that in the absence of periodontal treatment aimed at elimination of E. gingivalis, bacteria could exit the amoebae and result in refractory periodontitis. ${ }^{28}$

\section{E. gingivalis and immunocompromised patients:}

Lucht et al. conducted a study in HIV type-1 infected patients with periodontitis. They studied the association of $\mathrm{E}$. gingivalis with periodontal disease in HIV-1 infected patients. Thestudy results showed that the control group with E. gingivalis in plaque did not have any periodontal disease or gingivitis and had good oral hygiene, while all 10 HIV-1-infected patients with E. gingivalis in saliva/ plaque had periodontal disease. Periodontal disease was observed in $29 \%$ of the HIV-1 infected patients and $E$. gingivalis was present in $77 \%$ of these patients. E. gingivalis was not present in HIVlinfected patients without periodontal disease. Lucht et al. for the first time demonstrated that $E$. gingivalis can be involved in periodontal disease in HIV-1 infected patients. ${ }^{29} \mathrm{E}$. gingivalis was the only protozoa found in the oral cavities of HIV/1 infected patients with periodontal disease. ${ }^{30}$ Patients undergoing chemotherapy showed higher prevalence $(58 \%)$ of $\mathrm{E}$. gingivalis than the normal control $(34 \%){ }^{31}$

\section{E. gingivalis and peri-implantitis:}

Recently E. gingivalis was shown to be associated with peri-implantitis. Microscopic evaluation allowed visualizing the presence of amoeba in 31 out of 32 patients suffering from periimplantitis. Complete removal of $\mathrm{E}$. gingivalis from peri-implant sites is difficult if not impossible in the view of presence of persistent inflammation consisting of polymorphonuclear neutrophils providing a constant supply of food to the 
trophozoites. E. gingival is may phagocytose and lyse the PMNs and release cell contents like proteases especially cysteine proteases capable of degrading the laminin, fibronectin and collagen. ${ }^{10}$

\section{Challenges for determining the exact role of E. gingivalis in periodontal disease:}

It is extremely difficult to culture the organism and it cannot be cultured in the absence of bacteria. ${ }^{33}$ Some bacteria accompanying the xenic cultures are detrimental to growth of E. gingivalis while other bacteria are beneficial to its growth.

\section{Future considerations:}

Putative role of E. gingivalis in pathogenesis of periodontal disease could be determined by,

1. Using molecular biology techniques to identify virulence factors in clinical isolates

2. Identifying interactions between e. Gingivalis and periodontopathic bacteria

3. Identifying the genome of E. Gingivalis

\section{Conclusion:}

Periodontitis is a multifactorial chronic inflammatory disease. It is a reflection of interplay between multiple etiologic agents, host factors and environmental factors. Though the etiological role of $\mathrm{E}$. gingivalis in periodontitis has not been proved till date its presence in periodontal pockets and absence in healthy gingival sites shows that it is associated with the pathogenic process of periodontal disease. Evidence of greater association of $E$. gingivalis with periodontitis lesions in immunocompromised and HIV-1 infected patients indicates that periodontal treatment should also include measures aimed at elimination of $E$. gingivalis. While the association of the organism with periodontal disease was proved its role as etiologic agent can be proved only with the discovery of putative virulence factors and its interactions with periodontopathic bacteria. Development of molecular biologic techniques to detect virulence factors and interactions with periodontopathic bacteria might prove its role as periodontopathogen in future.

\section{References:}

1. Palmer RJ J r. Composition and development of oral bacterial communities. Perio 2000 2014; 64:20-39.

2. Bik EM, Long CD, Armitage GC, et al. Bacterial diversity in the oral cavity of 10 healthy individuals. ISME J 2010; 4:962-974.

3. Slots J. Herpesviral-bacterial interactions in periodontal diseases. Periodontal 2000 2010; 52:117-140.

4. Cappuyns I, Gugerli P, Mombelli A. Viruses in periodontal disease-a review. Oral Dis 2005; 11:219-229.

5. Slots J, Rams TE, Listgarten MA. Yeasts, enteric rods and pseudomonas in the subgingival flora of severe adult periodontitis. Oral Microbiol Immunol 1988; 3:47-52.

6. Vickerman MM, Brossard KA, Funk DB, J esionowski AM, Gill SR. Phylogenetic analysis of bacterial and archaeal species in symptomatic and asymptomatic endodontic infections. J Med Microbiol 2007; 56:110-118.

7. Lepp PW, Brinig M M, Ouverney CC, Palm K, Armitage GC, Relman DA. Methanogenic Archaea and human periodontal disease. Proc Natl Acad Sci U S A 2004; 101:6176-6181.

8. Norberg CMBM, Ribeiro PS, Carvalho NS, Sanches FG, de Carvalho RW, Norberg AN. Entamoeba gingivalis (Gros, 1849) and Trichomonas tenax (Muller, 1773) oral infections in patients from Baixada Fluminense, province of Rio de J aneiro, Brazil SJ PH 2014; 2(4):288-292.

9. Lyons T, Scholten T, Palmer J C, Stanfield E. Oral Amoebiasis: The Role of Entamoeba gingivalis in Periodontal Disease. Quintessence Int Dent Dig 1983; 14:1245-1248.

10. Bonner M, Amard V, Verdy, et al. Antiparasitic treatment of periodontitis and peri-implantitis: 12-months multicentric follow-up. Actual Odonto-Stomatol 2013; 261:22-28.

11. Wantland WW, Wantland EM. Incidence, Ecology, and Reproduction of Oral Protozoa. J Dent Res 1960; 39:863.

12. Ozcelík S, Gedík T, Gedík R, Malatyalp E. Investigation of the relationship between oral and dental health and presence of Entamoeba gingivalis and Trichomonas tenax. Turkiye Parazitol Derg 2010;34:155-159.

13. Keyes PH, Rams TE. A rationale for management of periodontal diseases: rapid identification of microbial 
'therapeutic targets' with phase-contrast microscopy. J Am Dent Assoc 1983; 106:803-812.

14. Dao AH, Robinson DP, Wong SW. F requency of Entamoeba gingivalis in human gingival scrapings. Am J Clin Pathol 1983; 80:380-383.

15. Derda M, Hadaœ E, Antczak E, Wojt WJ . Incidence of Entamoeba gingivalis in the oral cavity of students. J Stoma 2011; 64:784-795.

16. Albuquerque RLC J r, de Melo CM, de Santana WA, Ribeiro $J \mathrm{~L}$, Silva FA. Incidence of Entamoeba gingivalis and Trichomonas tenax in samples of dental biofilm and saliva from patients with periodontal disease. Rev Gaúcha Odontol 2011;59:35-40.

17. Al-Saeed WM, Mahmood HJ. Prevalence of Entamoeba gingivalis in dental patients in Mosul. Al-Rafidain Dent J 2001; 1:65-68.

18. Chen J F, Liu GY, Wen WR. Studies on the continuous culture and pathogenicity of Entamoeba gingivalis. Chin J Parasitol Parasitic Dis 2000; 18(2):84-86.

19. Bhaijee F, Bell D. Entamoeba gingivalis in Acute Osteomyelitis of the Mandible. Case Report Med 2011:357301.

20. Chen J F, Wen WR, Liu GY, Chen WL, Lin LG, Hong HY. Studies on periodontal disease caused by Entamoeba gingivalis and its pathogenetic mechanism. Rev China Med J 2001; 114(12):12-15.

21. Sarowska J, Wojnicz D, Kaczkowski H, J ankowski S. The occurrence of Entamoeba gingivalis and Trichomonas tenax in patients with periodontal disease. Adv Clin Exp Med 2004; 13(2):291-297.

22. Feki A, M olet B, Haag R, Kremer M. Protozoa of the human oral cavity (epidemiological correlations and pathogenic possibilities). J Biol Buccale 1981; 9:155-161.
23. Feki A, Molet B. Importance of Trichomonas tenax and Entamoeba gingivalis protozoa in the human oral cavity. Rev Odontostomatol 1990; 19:37-45.

24. El-Azzoumi M, El-Brady AMS. Frequency of Entamoeba gingivalis among periodontal and patients under chemotherapy. J Egypt Soc Parasitol 1994; 24:649-655.

25. Al-Saeed WM. Pathogenic effect of Entamoeba gingivalis on gingival tissues of rats. Al-Rafidain Dent J 2003; 3:7073.

26. Barrett MT. The protozoa of the mouth in relation to pyorrhea alveolaris. Dent Cosm 1914;56:948-953

27. Trim RD, Skinner MA, Farone MB, DuBois J D, Newsome $A L$. Use of PCR to detect Entamoeba gingivalis in diseased gingival pockets and demonstrate its absence in healthy gingival sites. Parasitol Res 2011; 109:857-864.

28. Derderian GM. An in vitro study of the co-cultivation of Actinobacillus actinomycetemcomitans. M.S.D Indiana University School of Dentistry, 1992.

29. Lucht E, Evengard B, Skott J, Pehrson P, Nord CE. Entamoeba gingivalis in Human Immunodeficiency Virus Type 1-Infected Patients with Periodontal Disease. Clinical Infectious Diseases 1998; 27:471-473.

30. Ghabanchi J , Zibaei M, Afkar MD, SarbazieAH. Prevalence of oral Entamoeba gingivalis and Trichomonas tenax in patients with periodontal disease and healthy population in Shiraz, southern Iran. Indian J Dent Res 2010; 21:8991.

31. Azzouni MZE, Badry AMSE. Frequency of Entamoeba gingivalis among periodontal and patients under chemotherapy. J Egypt Soc Parasitol 1994; 24:649-655.

32. Gannon J T, Linke HA. Synergistic growth studies of Entamoeba gingivalis using an Ecologen. Int J Parasitol 1992; 22:927-931.

\section{Gain quick access to our journal online View our journal at www.nacd.in}

